Article

\title{
Sharp Convex Bounds on the Aggregate Sums-An Alternative Proof
}

\author{
Chuancun Yin * and Dan Zhu
}

School of Statistics, Qufu Normal University, Qufu 273165, Shandong, China; zhudanspring@163.com

* Correspondence: ccyin@mail.qfnu.edu.cn; Tel.: +86-537-4453221

Academic Editor: Mogens Steffensen

Received: 8 May 2016; Accepted: 21 September 2016; Published: 29 September 2016

Abstract: It is well known that a random vector with given marginals is comonotonic if and only if
it has the largest convex sum, and that a random vector with given marginals (under an additional
condition) is mutually exclusive if and only if it has the minimal convex sum. This paper provides an
alternative proof of these two results using the theories of distortion risk measure and expected utility.

Keywords: comonotonicity; convex order; distortion risk measure; mutual exclusivity; stop-loss order

\section{Introduction}

After years of efforts made by researchers, the study of sharp convex bounds on the sum of random variables (also known as aggregate sums) with given marginal distributions but unknown dependence structure has achieved many significant results. Mathematically, given an arbitrary Fréchet space $\mathcal{R}\left(F_{1}, \ldots, F_{n}\right)$ of all random vectors having $F_{1}, \ldots, F_{n}$ as marginal distributions, the aim is to find two random vectors $\left(X_{1}^{m}, \ldots, X_{n}^{m}\right)$ and $\left(X_{1}^{M}, \ldots, X_{n}^{M}\right)$ belonging to $\mathcal{R}\left(F_{1}, \ldots, F_{n}\right)$, such that

$$
\sum_{i=1}^{n} X_{i}^{m} \leq_{c x} \sum_{i=1}^{n} X_{i} \leq_{c x} \sum_{i=1}^{n} X_{i}^{M}
$$

for any $\left(X_{1}, \ldots, X_{n}\right) \in \mathcal{R}\left(F_{1}, \ldots, F_{n}\right)$, where $\leq_{c x}$ denotes the convex order. By definition, for a pair of random variables $X$ and $Y$, we say that $X$ is less than $Y$ in the sense of convex order, denoted as $X \leq_{c x} Y$, if $E f(X) \leq E f(Y)$ for every convex function $f$, provided that expectations $E f(X)$ and $E f(Y)$ exist. In actuarial science, it is common to define convex order by using a stop-loss transform: $X \leq_{c x} Y \Leftrightarrow E X=E Y$ and $X \leq_{s l} Y$. Here $X$ is said to precede $Y$ in the stop-loss order sense, notation $X \leq_{s l} Y$, if and only if $X$ has lower stop-loss premiums than $Y$ :

$$
E(X-d)_{+} \leq E(Y-d)_{+},-\infty<d<\infty
$$

A summary of other characterizations and properties of convex order can be found, for example, in $[1,2]$.

Comonotonicity plays a crucial role in determining convex upper bound on aggregate sum. Let us recall the definition. For any $\mathbf{X} \in \mathcal{R}\left(F_{1}, \ldots, F_{n}\right), \mathbf{X}$ is said to be comonotonic if

$$
F_{\mathbf{X}}(\underline{x})=\min _{1 \leq k \leq n} F_{k}\left(x_{k}\right), \forall \underline{x}=\left(x_{1}, x_{2}, \ldots, x_{n}\right) \in \mathbb{R}^{n}
$$

Equivalently, $\mathbf{X}$ is comonotonic if and only if $\mathbf{X} \stackrel{d}{=}\left(F_{1}^{-1}(U), \ldots, F_{n}^{-1}(U)\right)$, where $U$ is a random variable uniformly distributed on the interval $[0,1]$, denoted as $U \sim U[0,1]$. The concept of comonotonicity was introduced by Yaari [3] and Schmeidler [4]. For more details and other characterizations about the concept of comonotonicity and its applications in actuarial science and 
finance, we refer to the overview papers by Dhaene et al. [5,6] and more recently in [7]. Let $S$ be the sum $X_{1}+\ldots+X_{n}$ and $S^{c}$ be the comonotonic sum $X_{1}^{c}+\ldots+X_{n}^{c}$, where $\left(X_{1}^{c}, \ldots, X_{n}^{c}\right)$ is the comonotonic counterpart of $\mathbf{X}=\left(X_{1}, \ldots, X_{n}\right)$. A well-known result between the sums $S$ and $S^{c}$ says that $S \leq_{c x} S^{c}$. Proofs of this fundamental result can be found in [8-12]. Müller [13] extended the result to higher dimensions as a special case of the concept of supermodular ordering. A simple geometric argument is given in [14], and Cheung [15] provided a new proof using the theory of majorization. The converse remains valid under the assumption that all marginal distribution functions are continuous and that the underlying probability space $(\Omega, \mathcal{F}, \mathcal{P})$ is atomless. For more details, see [16]. This continuity assumption on the marginals was removed by Cheung [17]. A simple proof without the assumption that the underlying probability space $(\Omega, \mathcal{F}, \mathcal{P})$ is atomless was given by Mao and $\mathrm{Hu}$ [18]. Some equivalent conditions on comonotonicity can be found in [19]. To summarize the above results, we arrive at the following theorem:

Theorem 1. If $\left(X_{1}^{*}, \ldots, X_{n}^{*}\right) \in \mathcal{R}\left(F_{1}, \ldots, F_{n}\right)$, then $\left(X_{1}^{*}, \ldots, X_{n}^{*}\right)$ is comonotonic if, and only if

$$
X_{1}+\ldots+X_{n} \leq_{c x} X_{1}^{*}+\ldots+X_{n}^{*} \text { for all }\left(X_{1}, \ldots, X_{n}\right) \in \mathcal{R}\left(F_{1}, \ldots, F_{n}\right) .
$$

Now we focus on the lower convex bound of $\mathcal{R}\left(F_{1}, \ldots, F_{n}\right)$. When $n=2$, the minimum sharp bound is obtained by the counter-monotonic scenario:

$$
F_{1}^{-1}(U)+F_{2}^{-1}(1-U) \leq_{c x} X_{1}+X_{2} \text { for any }\left(X_{1}, X_{2}\right) \in \mathcal{R}\left(F_{1}, F_{2}\right),
$$

where $U \sim U[0,1]$. Proofs for this assertion can be found in [9,19]. Moreover, Cheung and Lo [20] shows that the converse remains valid. However, the sharp lower convex bound for $n \geq 3$ is missing in general. Bernard et al. [21] gave an example showing that $\sum_{i=1}^{n} X_{i}$ does not have a sharp lower convex bound. Sufficient conditions for the existence of a such sharp lower convex bound for some classes of distributions can be found in $[19,21-24]$. In another special case, when $F_{1}, \ldots, F_{n}$ are defined on $[0, \infty)$ with $\sum_{i=1}^{n}\left(1-F_{i}(0)\right) \leq 1$, the convex lower bound is obtained by the mutually exclusive scenario:

$$
X_{1}^{*}+\ldots+X_{n}^{*} \leq_{c x} X_{1}+\ldots+X_{n}
$$

for any $\left(X_{1}, \ldots, X_{n}\right) \in \mathcal{R}\left(F_{1}, \ldots, F_{n}\right)$, where $\left(X_{1}^{*}, \ldots, X_{n}^{*}\right) \in \mathcal{R}\left(F_{1}, \ldots, F_{n}\right)$ and $P\left(X_{i}^{*}>0, X_{j}^{*}>0\right)=0$ for all $i \neq j$ (see $[25,26])$. Mutual exclusivity can be considered as the strongest negative dependence structure in a multivariate setting. It was first studied in [26] when the marginals $F_{1}, F_{2}, \ldots, F_{n}$ are two-point distributions and in Dhaene and Denuit [25] in a more general setting. A revisited and further characterized treatment of mutual exclusivity can be found in [27]. A recent overview paper by Puccetti and Wang [19] introduced the concept of pairwise countermonotonicity, which is more general than that of mutual exclusivity. Moreover, several equivalent conditions on pairwise countermonotonicity have been provided (see Theorem 3.3 in [19]).

Definition 1. (Definition 3.4 in [27]) Let $X_{1}, \ldots, X_{n}$ be random variables with essential infima $l_{1}, \ldots, l_{n}$ and essential suprema $u_{1}, \ldots, u_{n}$, respectively. They are said to be

(i) mutually exclusive from below if $P\left(X_{i}>l_{i}, X_{j}>l_{j}\right)=0$ for all $i \neq j$;

(ii) mutually exclusive from above if $P\left(X_{i}<u_{i}, X_{j}<u_{j}\right)=0$ for all $i \neq j$.

The following theorem is concerned with mutually exclusive random variables and the minimal lower bound in convex order. 
Theorem 2. (Theorem 5.1 in [27]) Let $\mathbf{X}^{*}=\left(X_{1}^{*}, \ldots, X_{n}^{*}\right)$ be a fixed random vector in $\mathcal{R}\left(F_{1}, \ldots, F_{n}\right)(n \geq 3)$ which satisfies $\sum_{i=1}^{n}\left(1-F_{i}\left(l_{i}\right)\right) \leq 1$ or $\sum_{i=1}^{n} F_{i}\left(u_{i}-\right) \leq 1$. Then $\mathbf{X}^{*}$ is mutually exclusive if, and only if

$$
X_{1}^{*}+\ldots+X_{n}^{*} \leq_{c x} X_{1}+\ldots+X_{n}
$$

for all $\left(X_{1}, \ldots, X_{n}\right) \in \mathcal{R}\left(F_{1}, \ldots, F_{n}\right)$.

In this short note, we give alternative proofs of Theorems 1 and 2 in the next two sections.

\section{Proof of Theorem 1}

To prove Theorem 1, we need two useful lemmas. Here are some notations. Let $F_{X}$ be the cumulative distribution function of random variable $X$, and the decumulative distribution function is denoted by $\bar{F}_{X}$; i.e., $\bar{F}_{X}(x)=1-F_{X}(x)=P(X>x)$. A distortion function is defined as a non-decreasing function $g:[0,1] \rightarrow[0,1]$ such that $g(0)=0$ and $g(1)=1$. The distortion risk measure associated with distortion function $g$ is defined by

$$
\rho_{g}[X]=\int_{0}^{+\infty} g\left(\bar{F}_{X}(x)\right) d x+\int_{-\infty}^{0}\left[g\left(\bar{F}_{X}(x)\right)-1\right] d x,
$$

for any random variable $X$, provided at least one of the two integrals above is finite. If $X$ is a non-negative random variable, then $\rho_{g}$ reduces to

$$
\rho_{g}[X]=\int_{0}^{+\infty} g\left(\bar{F}_{X}(x)\right) d x .
$$

Obviously, a concave distortion function is continuous on $(0,1]$ and can only jump at 0 . In view of Theorem 6 of Dhaene et al. [28], we know that for any concave distortion function $g$, one can rewrite $\rho_{g}[X]$ as

$$
\rho_{g}[X]=\int_{[0,1]} \operatorname{VaR}_{1-q}[X] d g(q),
$$

where $\operatorname{VaR}_{p}[X]$ denotes the value-at-risk at level $p$ of $X$ and is defined as

$$
\operatorname{VaR}_{p}[X]=\inf \left\{x \in \mathbb{R} \mid F_{X}(x) \geq p\right\}, p \in(0,1) .
$$

The following theorem shows that stop-loss order can be characterized in terms of ordered concave distortion risk measures (see $[29,30])$. Here we provide a short proof.

Lemma 1. For any random vector $(X, Y)$, we have that $X \leq_{s l} Y$ if and only if their respective concave distortion risk measures are ordered: $X \leq_{s l} Y \Leftrightarrow \rho_{g}[X] \leq \rho_{g}[Y]$ for all concave distortion functions $g$. In particular, if $E[X]=E[Y]$, then $X \leq_{c x} Y \Leftrightarrow \rho_{g}[X] \leq \rho_{g}[Y]$ for all concave distortion functions $g$.

Proof. For any concave distortion function $g$, it is differentiable at all but at most countably many points on $[0,1]$. The distortion risk measure $\rho_{g}$ can be written as

$$
\rho_{g}[X]=\int_{0}^{1} \operatorname{TVaR}_{p}[X] d \mu(p)
$$

where $\mu(p):=\int_{0}^{p}(1-\alpha) d v(\alpha)$ is a probability measure in which $v$ is defined by $v([0, p])=g^{\prime}(1-p)$, $\mathrm{TVaR}_{p}$ is the tail value-at-risk(also known as the expected shortfall), defined as

$$
\operatorname{TVaR}_{p}[X]=\frac{1}{1-p} \int_{p}^{1} \operatorname{VaR}_{w}[X] d w, p \in(0,1)
$$


which is a distortion risk measure corresponding to the concave distortion function

$$
g(x)=\min \left\{\frac{x}{1-p}, 1\right\}, 0<p<1 .
$$

The result follows as $X \leq_{s l} Y \Leftrightarrow \operatorname{TVaR}_{p}[X] \leq \operatorname{TVaR}_{p}[Y]$ for all $p \in(0,1)$ (see Theorem 3.2 in [30]).

The following subadditivity theorem can be found in [29], the bivariate case can be found in [31], see also [32].

Lemma 2. For any concave distortion function $g$ and $\left(X_{1}, \ldots, X_{n}\right) \in \mathcal{R}\left(F_{1}, \ldots, F_{n}\right)$, we have

$$
\rho_{g}\left[X_{1}+\ldots+X_{n}\right] \leq \rho_{g}\left[X_{1}\right]+\ldots+\rho_{g}\left[X_{n}\right] .
$$

Proof of Theorem 1. First we assume $\left(X_{1}^{*}, \ldots, X_{n}^{*}\right) \in \mathcal{R}\left(F_{1}, \ldots, F_{n}\right)$ is comonotonic. For any concave distortion function $g$ and $\left(X_{1}, \ldots, X_{n}\right) \in \mathcal{R}\left(F_{1}, \ldots, F_{n}\right)$, by Lemma 2 we have

$$
\rho_{g}\left[X_{1}+\ldots+X_{n}\right] \leq \rho_{g}\left[X_{1}\right]+\ldots+\rho_{g}\left[X_{n}\right]
$$

Comonotonicity of $\left(X_{1}^{*}, \ldots, X_{n}^{*}\right) \in \mathcal{R}\left(F_{1}, \ldots, F_{n}\right)$ implies that (cf. Dhaene et al. [30])

$$
\rho_{g}\left[X_{1}\right]+\ldots+\rho_{g}\left[X_{n}\right]=\rho_{g}\left[X_{1}^{*}+\ldots+X_{n}^{*}\right] .
$$

Therefore, combining (1) with (2), one has

$$
\rho_{g}\left[X_{1}+\ldots+X_{n}\right] \leq \rho_{g}\left[X_{1}^{*}+\ldots+X_{n}^{*}\right],
$$

and the desired result follows from Lemma 1.

To prove the other implication, we assume that $\left(X_{1}^{*}, \ldots, X_{n}^{*}\right) \in \mathcal{R}\left(F_{1}, \ldots, F_{n}\right)$ and

$$
X_{1}+\ldots+X_{n} \leq_{c x} X_{1}^{*}+\ldots+X_{n}^{*} \text { for all }\left(X_{1}, \ldots, X_{n}\right) \in \mathcal{R}\left(F_{1}, \ldots, F_{n}\right) .
$$

From Lemma 1, we have that

$$
\rho_{g}\left[X_{1}+\ldots+X_{n}\right] \leq \rho_{g}\left[X_{1}^{*}+\ldots+X_{n}^{*}\right],
$$

for any concave distortion function $g$. In particular,

$$
\rho_{g}\left[X_{1}^{c}+\ldots+X_{n}^{c}\right] \leq \rho_{g}\left[X_{1}^{*}+\ldots+X_{n}^{*}\right]
$$

where $\left(X_{1}^{c}, \ldots, X_{n}^{c}\right)$ is the comonotonic counterpart of $\left(X_{1}, \ldots, X_{n}\right)$. On the other hand, by Lemma 2, we get

$$
\rho_{g}\left[X_{1}^{*}+\ldots+X_{n}^{*}\right] \leq \rho_{g}\left[X_{1}^{*}\right]+\ldots+\rho_{g}\left[X_{n}^{*}\right]
$$

Note that

$$
\rho_{g}\left[X_{1}^{c}+\ldots+X_{n}^{c}\right]=\rho_{g}\left[X_{1}^{c}\right]+\ldots+\rho_{g}\left[X_{n}^{c}\right]
$$

and

$$
\rho_{g}\left[X_{1}^{c}\right]+\ldots+\rho_{g}\left[X_{n}^{c}\right]=\rho_{g}\left[X_{1}^{*}\right]+\ldots+\rho_{g}\left[X_{n}^{*}\right] .
$$

It follows from (3)-(6) that we have

$$
\rho_{g}\left[X_{1}^{c}+\ldots+X_{n}^{c}\right]=\rho_{g}\left[X_{1}^{*}+\ldots+X_{n}^{*}\right],
$$


for any concave distortion function $g$. Therefore, Theorem 7 in [17] implies $\left(X_{1}^{*}, \ldots, X_{n}^{*}\right)$ is comonotonic. This ends the proof of Theorem 1.

\section{Proof of Theorem 2}

To prove Theorem 2, we need two useful lemmas. Lemma 3 gives a necessary and sufficient condition for the convex order of two random variables.

Lemma 3. (Proposition 3.4.3 in [1]) Given two ros X and $Y$, then the following statements are equivalent:

(1) $X \leq_{c x} Y$.

(2) $E[v(X)] \leq E[v(Y)]$ for all convex functions $v$, such that the expectations exist.

(3) $E[v(X)] \leq E[v(Y)]$ for all functions $v$ with $v^{\prime \prime} \geq 0$, such that the expectations exist.

The following lemma, due to Cheung and Lo [33], will play a crucial role in the proof of Theorem 2.

Lemma 4. (Theorem 3.1 in [33]) Let $X_{1}, \ldots, X_{n}$ be non-negative random variables, and $f$ be a convex function such that $E\left[f\left(\sum_{i=1}^{n} X_{i}\right)\right]$ exists.

(i) We have

$$
E\left[f\left(\sum_{i=1}^{n} X_{i}\right)\right] \geq \sum_{i=1}^{n} E\left[f\left(X_{i}\right)\right]-(n-1) f(0) ;
$$

(ii) if $f$ is strictly convex, then

$$
E\left[f\left(\sum_{i=1}^{n} X_{i}\right)\right]=\sum_{i=1}^{n} E\left[f\left(X_{i}\right)\right]-(n-1) f(0)
$$

if, and only if $X_{1}, \ldots, X_{n}$ are mutually exclusive random variables in the sense of Dhaene and Denuit [25].

Remark 1. We remark that the "if part" is still true when the function $f$ is convex, but not necessarily strictly convex.

Proof of Theorem 2. To prove Theorem 2, as in the proof to Lemma 3.6 in [27], there are three cases to consider. Recall that $l_{1}, \ldots, l_{n}$ are the essential infima of random variables $X_{1}, \ldots, X_{n}$, respectively (see Definition 1).

Case $1 . l_{1}=\ldots=l_{n}=0$. We assume $\left(X_{1}^{*}, \ldots, X_{n}^{*}\right) \in \mathcal{R}\left(F_{1}, \ldots, F_{n}\right)$ is mutually exclusive. For any convex function $u$ and $\left(X_{1}, \ldots, X_{n}\right) \in \mathcal{R}\left(F_{1}, \ldots, F_{n}\right)$, by Lemma 4 (i) we have

$$
E\left[u\left(\sum_{i=1}^{n} X_{i}\right)\right] \geq \sum_{i=1}^{n} E\left[u\left(X_{i}\right)\right]-(n-1) u(0) .
$$

Thanks to Lemma 4 (ii) and Remark 1, mutual exclusivity of $\left(X_{1}^{*}, \ldots, X_{n}^{*}\right)$ implies that

$$
E\left[u\left(\sum_{i=1}^{n} X_{i}^{*}\right)\right]=\sum_{i=1}^{n} E\left[u\left(X_{i}^{*}\right)\right]-(n-1) u(0) .
$$

Therefore, combining (8) with (9), and noting that $E\left[u\left(X_{1}^{*}\right)\right]+\ldots+E\left[u\left(X_{n}^{*}\right)\right]=E\left[u\left(X_{1}\right)\right]+\ldots+$ $E\left[u\left(X_{n}\right)\right]$, one has

$$
E\left[u\left(\sum_{i=1}^{n} X_{i}^{*}\right)\right] \leq E\left[u\left(\sum_{i=1}^{n} X_{i}\right)\right],
$$

from which and from Lemma 3, we deduce that

$$
X_{1}^{*}+\ldots+X_{n}^{*} \leq_{c x} X_{1}+\ldots+X_{n}
$$


for all $\left(X_{1}, \ldots, X_{n}\right) \in \mathcal{R}\left(F_{1}, \ldots, F_{n}\right)$.

To prove the other implication, we assume that $\left(X_{1}^{*}, \ldots, X_{n}^{*}\right) \in \mathcal{R}\left(F_{1}, \ldots, F_{n}\right)$ and

$$
X_{1}^{*}+\ldots+X_{n}^{*} \leq_{c x} X_{1}+\ldots+X_{n} \text { for all }\left(X_{1}, \ldots, X_{n}\right) \in \mathcal{R}\left(F_{1}, \ldots, F_{n}\right) .
$$

From Lemma 3, we have that

$$
E\left[u\left(X_{1}^{*}+\ldots+X_{n}^{*}\right)\right] \leq E\left[u\left(X_{1}+\ldots+X_{n}\right)\right]
$$

for all convex functions $u$. In particular,

$$
E\left[u\left(X_{1}^{*}+\ldots+X_{n}^{*}\right)\right] \leq E\left[u\left(X_{1}^{M}+\ldots+X_{n}^{M}\right)\right]
$$

where $\left(X_{1}^{M}, \ldots, X_{n}^{M}\right)$ is the mutually exclusive counterpart of $\left(X_{1}, \ldots, X_{n}\right)$. On the other hand, by Lemma 4 and Remark 1, we get

$$
\left.E\left[u\left(X_{1}^{*}+\ldots+X_{n}^{*}\right)\right] \geq E\left[u\left(X_{1}^{*}\right)\right]+\ldots+E\left[u\left(X_{n}^{*}\right)\right)\right]-(n-1) u(0),
$$

and

$$
\left.E\left[u\left(X_{1}^{M}+\ldots+X_{n}^{M}\right)\right]=E\left[u\left(X_{1}^{M}\right)\right]+\ldots+E\left[u\left(X_{n}^{M}\right)\right)\right]-(n-1) u(0) .
$$

If $\left(X_{1}^{*}, \ldots, X_{n}^{*}\right)$ is not mutually exclusive, then, for strict convex $u$,

$$
\left.E\left[u\left(X_{1}^{*}+\ldots+X_{n}^{*}\right)\right] \neq E\left[u\left(X_{1}^{*}\right)\right]+\ldots+E\left[u\left(X_{n}^{*}\right)\right)\right]-(n-1) u(0) .
$$

Combining (10)-(12) with (13), we get

$$
\left.\left.E\left[u\left(X_{1}^{*}\right)\right]+\ldots+E\left[u\left(X_{n}^{*}\right)\right)\right]<E\left[u\left(X_{1}^{M}\right)\right]+\ldots+E\left[u\left(X_{n}^{M}\right)\right)\right],
$$

for any strict convex function $u$. This contradicts $\left(X_{1}^{*}, \ldots, X_{n}^{*}\right)$ and $\left(X_{1}^{M}, \ldots, X_{n}^{M}\right)$ having the same marginals. Thus, $\left(X_{1}^{*}, \ldots, X_{n}^{*}\right)$ is mutually exclusive.

Case 2. $\left(X_{1}^{*}, \ldots, X_{n}^{*}\right)$ is mutually exclusive from below. For any $\left(X_{1}, \ldots, X_{n}\right) \in \mathcal{R}\left(F_{1}, \ldots, F_{n}\right)$, then $Z:=X_{i}-l_{i}$ are non-negative random variables, and $Z^{*}:=X_{i}^{*}-l_{i}$ are non-negative mutually exclusive random variables. Applying the result in Case 1 , we obtain that $\left(X_{1}^{*}, \ldots, X_{n}^{*}\right)$ is mutually exclusive $\Leftrightarrow$ $\left(X_{1}^{*}-l_{1}, \ldots, X_{n}^{*}-l_{n}\right)$ is mutually exclusive

$$
\begin{gathered}
\Leftrightarrow \sum_{i=1}^{n}\left(X_{i}^{*}-l_{i}\right) \leq_{c x} \sum_{i=1}^{n}\left(X_{i}-l_{i}\right) \Leftrightarrow \sum_{i=1}^{n} X_{i}^{*}-\sum_{i=1}^{n} l_{i} \leq_{c x} \sum_{i=1}^{n} X_{i}-\sum_{i=1}^{n} l_{i} \\
\Leftrightarrow \sum_{i=1}^{n} X_{i}^{*} \leq_{c x} \sum_{i=1}^{n} X_{i} .
\end{gathered}
$$

Case 3. $\left(X_{1}^{*}, \ldots, X_{n}^{*}\right)$ is mutually exclusive from above. For any $\left(X_{1}, \ldots, X_{n}\right) \in \mathcal{R}\left(F_{1}, \ldots, F_{n}\right)$, applying the result in Case 2 , we have $\left(X_{1}^{*}, \ldots, X_{n}^{*}\right)$ is mutually exclusive from above $\Leftrightarrow\left(-X_{1}^{*}, \ldots,-X_{n}^{*}\right)$ is mutually exclusive from below $\Leftrightarrow-\sum_{i=1}^{n} X_{i}^{*} \leq_{c x}-\sum_{i=1}^{n} X_{i} \Leftrightarrow \sum_{i=1}^{n} X_{i}^{*} \leq_{c x} \sum_{i=1}^{n} X_{i}$. The proof of Theorem 2 is now complete.

Acknowledgments: We are greatly indebted to Ruodu Wang and two anonymous referees for their insightful comments. The research was supported by the National Natural Science Foundation of China (No. 11171179, 11571198) and the Research Fund for the Doctoral Program of Higher Education of China (No. 20133705110002).

Author Contributions: These authors contributed equally to this work.

Conflicts of Interest: The authors declare no conflict of interest. 


\section{References}

1. Denuit, M.; Dhaene, J.; Goovaerts, M.; Kaas, R. Actuarial Theory for Dependent Risks: Measures, Orders and Models; John Wiley \& Sons: Chichester, UK, 2005.

2. Shaked, M.; Shanthikumar, J.G. Stochastic Orders; Springer: New York, NY, USA, 2007.

3. Yaari, M.E. The dual theory of choice under risk. Econometrica 1987, 55, 95-115.

4. Schmeidler, D. Integral representation without additivity. Proc. Am. Math. Soc. 1986, 97, 255-261.

5. Dhaene, J.; Denuit, M.; Goovaerts, M.J.; Kaas, R.; Vyncke, D. The concept of comonotonicity in actuarial science and finance: Theory. Insur. Math. Econ. 2002, 31, 3-33.

6. Dhaene, J.; Denuit, M.; Goovaerts, M.J.; Kaas, R.; Vyncke, D. The concept of comonotonicity in actuarial science and finance: Applications. Insur. Math. Econ. 2002, 31, 133-161.

7. Deelstra, G.; Dhaene, J.; Vanmaele, M. An overview of comonotonicity and its applications in finance and insurance. In Advanced Mathematical Methods for Finance; Oksendal, B., Nunno, G., Eds.; Springer: Heidelberg, Germany, 2010; pp. 155-179.

8. Meilijson, I.; Nadas, A. Convex majorization with an application to the length of critical paths. J. Appl. Probab. 1979, 16, 671-677.

9. Tchen, A.H. Inequalities for distributions with given marginals. Ann. Probab. 1980, 8, 814-827.

10. Rus̈chendorf, L. Solution of a statistical optimization problem by rearrangement methods. Metrika 1983, 30, 55-61.

11. Dhaene, J.; Goovaerts, M.J. Dependency of risks and stop-loss order. ASTIN Bull. 1996, 26, 201-212.

12. Dhaene, J.; Goovaerts, M.J. On the dependency of risks in the individual life model. Insur. Math. Econ. 1997, 19, 243-253.

13. Müller, A. Stop-loss order for portfolios of dependent risks. Insur. Math. Econ. 1997, 21, $219-223$.

14. Kaas, R.; Dhaene, J.; Vyncke, D.; Goovaerts, M.; Denuit, M. A simple geometric proof that comonotonic risks have the convex-largest sum. ASTIN Bull. 2002, 32, 71-80.

15. Cheung, K.C. Comonotonic convex upper bound and majorization. Insur. Math. Econ. 2010, 47, 154-158.

16. Cheung, K.C. Characterization of comonotonicity using convex order. Insur. Math. Econ. 2008, 43, $403-406$.

17. Cheung, K.C. Characterizing a comonotonic random vector by the distribution of the sum of its components. Insur. Math. Econ. 2010, 47, 130-136.

18. Mao, T.T.; Hu, T.Z. A new proof of Cheung's characterization of comonotonicity. Insur. Math. Econ. 2011, 48, 214-216.

19. Puccetti, G.; Ruodu Wang, R. Extremal dependence concepts. Stat. Sci. 2015, 30, 485-517.

20. Cheung, K.C.; Lo, A. Characterizations of counter-monotonicity and upper comonotonicity by (tail) convex order. Insur. Math. Econ. 2013, 53, 334-342.

21. Bernard, C.; Jiang, X.; Wang, R. Risk aggregation with dependence uncertainty. Insur. Math. Econ. 2014, 54, 93-108.

22. Wang, B.; Wang, R. The complete mixability and convex minimization problems for monotone marginal distributions. J. Multivar. Anal. 2011, 102, 1344-1360.

23. Wang, B.; Wang, R. Joint mixability. Math. Op. Res. 2016, 41, 808-826.

24. Jakobsons, E.; Han, X.; Wang, R. General convex order on risk aggregation. Scand. Actuar. J. 2016, 8, 713-740.

25. Dhaene, J.; Denuit, M. The safest dependence structure among risks. Insur. Math. Econ. 1999, 25, 11-21.

26. Hu, T.Z.; Wang, Z.Q. On the dependence of risks and the stop-loss premiums. Insur. Math. Econ. 1999, 24, 323-332.

27. Cheung, K.C.; Lo, A. Characterizing mutual exclusivity as the strongest negative multivariate dependence structure. Insur. Math. Econ. 2014, 55, 180-190.

28. Dhaene, J.; Kukush, A.; Linders, D.; Tang, Q. Remarks on quantiles and distortion risk measures. Eur. Actuar. J. 2012, 2, 319-328.

29. Dhaene, J.; Wang, S.; Young, V.; Goovaerts, M. Comonotonicity and maximal stop-loss premiums. Bull. Swiss Assoc. Actuar. 2000, 2, 99-113.

30. Dhaene, J.; Vanduffel, S.; Tang, Q.; Goovaerts, M.; Kaas, R.; Vyncke, D. Risk measures and comonotonicity: A review. Stoch. Models 2006, 22, 573-606.

31. Denneberg, D. Non-Additive Measure and Integral; Kluwer Academic Publilshers: Dordrecht, The Netherlands, 1994. 
32. Wang, S.; Dhaene, J. Comonotonicity, correlation order and premium principles. Insur. Math. Econ. 1998, 22, 235-242.

33. Cheung, K.C.; Lo, A. General lower bounds on convex functionals of aggregate sums. Insur. Math. Econ. 2013, 53, 884-896. 